\title{
DIAGRAMAS DE ELLINGHAM E DE VAN'T HOFF: ALGUMAS CONSIDERAÇÕES
}

José de Alencar Simoni e Aécio Pereira Chagas*

Instituto de Química, Universidade Estadual de Campinas, CP 6154, 13084-971 Campinas - SP, Brasil

Recebido em 14/3/06; aceito em 14/6/06; publicado na web em 19/1/07

\begin{abstract}
ELLINGHAM AND VAN'T HOFF DIAGRAMS: SOME CONSIDERATIONS. The main subject of this article is to show the parallelism betwen the Ellingham and Van't Hoff diagrams. The first one is a graphic representation of the changes in the standard Gibbs free energy $\left(\Delta_{\mathrm{r}} G^{\theta}\right)$ as a function of $T$ and was introduced by Ellingham in 1944, in order to study metallurgic processes involving oxides and sulphides. On the other hand, the Van't Hoff diagram is a representation of the function $\ln K$ versus $(1 / \mathrm{T})$. The equivalence between both diagrams is easily demonstrated, making simple mathematical manipulations. In order to show the parallelism between both diagrams, they are presented briefly and two examples are discussed. The comparison of the both diagrams surely will be helpful to students and teachers in their learning and teaching activities, and will certainly enrich important aspects of chemical thermodynamics.
\end{abstract}

Keywords: Ellingham diagram; Van't Hoff diagram; chemical thermodynamics.

Os diagramas de Ellingham, ou seja, representações gráficas das funções $\Delta_{\mathrm{r}} G(T)$, foram introduzidos por H. J. T. Ellingham, em 1944, para estudo de processos metalúrgicos envolvendo óxidos e sulfetos ${ }^{1}$. Foram, posteriormente, difundidos por Richardson, porém seu uso ficou restrito à área metalúrgica².

Recentemente, livros mais gerais ${ }^{3-5}$ têm apresentados estes diagramas, inclusive para nitretos ${ }^{6} \mathrm{e}$ haletos metálicos ${ }^{7}$. Usos não metalúrgicos são mais raros, porém, recentemente Maia e Osório ${ }^{8}$ relataram uma aplicação. A Figura 1 traz um diagrama de Ellingham para óxidos 5 .

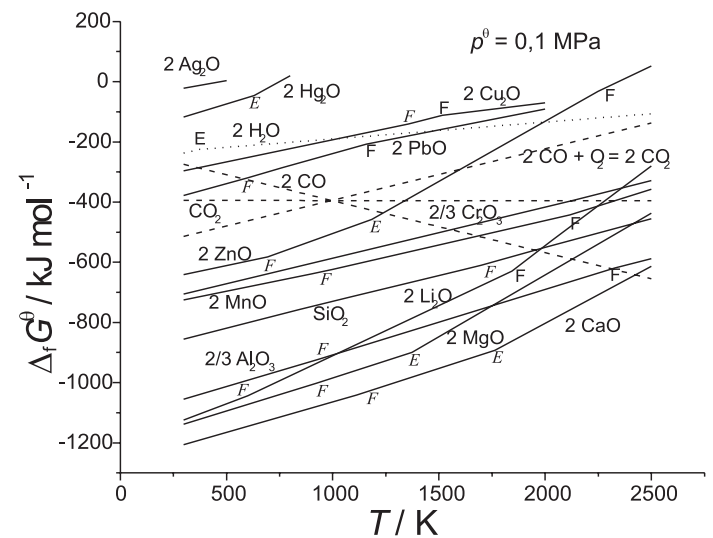

Figura 1. Diagrama de Ellingham para alguns óxidos. A energia livre de Gibbs de formação $\left(\Delta_{f} \mathrm{G}^{\theta}\right)$ para alguns óxidos está grafada em função da temperatura. O diagrama é normalizado para um mol de $O_{2}(g)$. A $\Delta_{f} \mathrm{G}^{\theta}(T) d a$ reação $2 \mathrm{CO}(\mathrm{g})+\mathrm{O}_{2}(\mathrm{~g})=2 \mathrm{CO}_{2}(\mathrm{~g})$ está também representada. Pode-se observar algumas inflexões nas retas, devido à mudança de fase nos óxidos ou nos elementos. As letras $\mathrm{F} e \mathrm{E}$ indicam fusão e ebulição dos elementos, respectivamente; F e E referem-se ao óxido. Para facilitar a visualização, indicou-se, no caso dos óxidos de carbono, por linhas tracejadas e, no caso da água, por linha pontilhada. Note que, dentre as espécies representadas, a única com coeficiente angular negativo é o $C O(g)$, daí a sua importância como redutor metalúrgico. Adaptado da ref. 5

*e-mail: aecio@iqm.unicamp.br
As funções $\Delta G(T)$ são praticamente lineares em relação à $T$, ou melhor, quando representadas graficamente em um diagrama de coordenadas $\Delta_{\mathrm{r}} G$ em função de $T$, são retas quebradas em que as inflexões, muitas vezes pouco acentuadas, são devidas às mudanças de fases (fusão, vaporização etc.) de um dos componentes. A função $\Delta_{r} G(T)$ tem a forma de uma das equações fundamentais da Termodinâmica Química:

$\Delta_{\mathrm{r}} G(T)=\Delta_{\mathrm{r}} H(T)-\Delta_{\mathrm{r}} S(T) T(p$ constante $)$

sendo $\Delta_{\mathrm{r}} H(T)$ o coeficiente linear e $\Delta_{\mathrm{r}} S(T)$ o coeficiente angular (o significado dos símbolos encontra-se no final do artigo). A rigor, as grandezas $H$ e $S$ são também funções de $T$, conforme indicado, porém elas são praticamente constantes com a mesma, fazendo com que $\Delta_{\mathrm{r}} G(T)$ se torne, portanto, praticamente linear. Esta independência de $H$ e $S$ em relação a $T$ é devida ao fato de $\Delta_{\mathrm{r}} C p(T)$ ser pequeno. Explicitando melhor, os valores de $H$ e $S$ a uma dada temperatura $T$ são dados, respectivamente, pelas equações:

$$
\begin{aligned}
& \Delta_{\mathrm{r}} H(T)=\Delta_{\mathrm{r}} H\left(T_{r}\right)+\int_{T_{r}}^{T} \Delta_{\mathrm{r}} C_{p}(T) \mathrm{d} T=\Delta_{\mathrm{r}} H\left(T_{r}\right)+\operatorname{Int} H(T) \quad(p \text { constante }) \\
& \Delta_{\mathrm{r}} S(T)=\Delta_{\mathrm{r}} S\left(T_{r}\right)+\int_{T_{r}}^{T}\left[\Delta_{\mathrm{r}} C_{p}(T) / T\right] \mathrm{d} T=\Delta_{\mathrm{r}} S\left(T_{r}\right)+\operatorname{Int} S(T) \quad(p \text { constante })
\end{aligned}
$$

em que $T_{r}$ é a temperatura de referência, usualmente $298,15 \mathrm{~K}$, e a notação das integrais foi simplificada ${ }^{5,9}$.

Como os valores das integrais acima são pequenos em relação aos outros dois respectivos termos do segundo membro, $\Delta_{\mathrm{r}} H\left(T_{r}\right)$ e $\Delta_{\mathrm{r}} S\left(T_{r}\right)$, praticamente $\Delta_{\mathrm{r}} H(T)$ e $\Delta_{\mathrm{r}} S(T)$ não variam. Como exemplo, tem-se o sistema representado pela equação química:

$\mathrm{Ti}(\mathrm{s})+\mathrm{O}_{2}(\mathrm{~g})=\mathrm{TiO}_{2}(\mathrm{~s}$, rutilo $)\left(p=p^{\theta}=0,1 \mathrm{MPa}\right)$

Será considerada a faixa de 298 a $1100 \mathrm{~K}$, larga o suficiente para se verificar as variações de cada termo e sem nenhuma mudança de fase dos componentes. A Tabela 1 apresenta os valores dos termos das Equações 2 e 3 em algumas temperaturas. Na Figura 2 tem-se um gráfico mostrando a variação da Int $S(T)$ (Equação 3) e 
de $\Delta_{r} S(T)$ com a $T$. Observar que $\Delta_{r} S(T)$ é dezenas de vezes maior (em valor absoluto) que a respectiva integral. Na Figura 3 tem-se a variação de Int $H(T)$ (Equação 2), $\Delta_{\mathrm{r}} H(T), \Delta_{\mathrm{r}} G(T)$ e $\Delta_{\mathrm{r}} S(T) T$ com a $T$. Observar que $\Delta_{\mathrm{r}} H(T)$ é centenas de vezes maior (em valor absoluto) que Int $H(T)$, além da linearidade dos outros termos. Nestes cálculos utilizaram-se as tabelas JANAF ${ }^{10}$.

Tabela 1. Valores das funções termodinâmicas (Eqs. 2 e 3) para a formação do dióxido de titânio a algumas temperaturas e a 0,1 MPa

\begin{tabular}{lccc}
\hline$T / \mathrm{K}$ & 298,15 & 700 & 1100 \\
\hline$\Delta_{\mathrm{r}} H^{\theta}(T) / \mathrm{kJ} \mathrm{mol}^{-1}$ & $-944,75$ & $-941,84$ & $-938,34$ \\
Int $H(T) / \mathrm{kJ} \mathrm{mol}^{-1}$ & 0 & 2,91 & 6,41 \\
$\Delta_{\mathrm{r}} S^{\theta}(T) / \mathrm{J} \mathrm{K}^{-1} \mathrm{~mol}^{-1}$ & $-185,62$ & $-180,00$ & $-175,94$ \\
$\mathrm{Int}^{\theta} S^{\theta}(T) / \mathrm{J} \mathrm{K}^{-1} \mathrm{~mol}^{-1}$ & 0 & 5,65 & 9,67 \\
$\Delta_{\mathrm{r}} S^{\theta}(T) T / \mathrm{kJ} \mathrm{mol}^{-1}$ & $-55,34$ & $-125,97$ & $-193,54$ \\
$\Delta_{\mathrm{r}} G^{\theta}(T) / \mathrm{kJ} \mathrm{mol}^{-1}$ & $-889,41$ & $-815,87$ & $-744,80$ \\
\hline
\end{tabular}

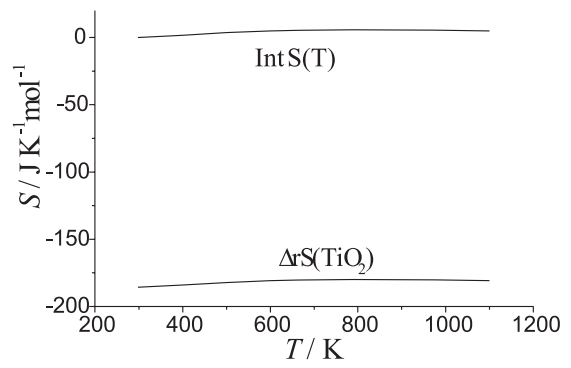

Figura 2. Variação da Int $S(T)$ e de $\Delta r S(T)$ com a $T$ (ver texto e Equação 3). Observar que $\Delta r S(T)$ é dezenas de vezes maior que a respectiva integral

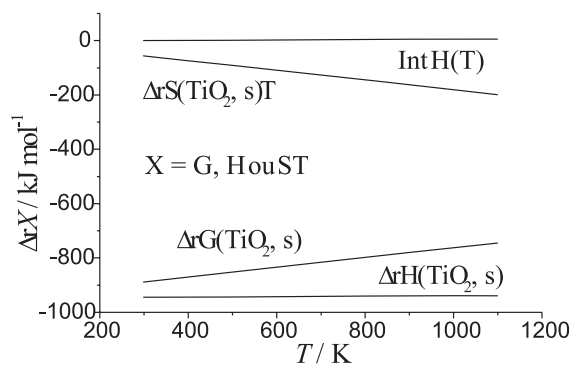

Figura 3. Variação de Int $H(T), \Delta r H(T), \Delta r G(T)$ e $\Delta r S(T) T$ com a $T$ (ver texto e Equações 1 e 2). Observar que $\Delta r H(T)$ é centenas de vezes maior que Int $H(T)$, além da linearidade dos outros termos

Da literatura, de modo geral, capta-se a impressão de que os diagramas de Ellingham só funcionam para substâncias que interessam à metalurgia (óxidos, sulfetos etc.), mas, como será mostrado a seguir, estes diagramas são de uso geral, como os de Van't Hoff, que são equivalentes.

Os diagramas de Van't Hoff são representações da função $\ln K$ $(1 / T)$, obtidas da integração da conhecida equação do mesmo nome ${ }^{5,9,11}$ :

$\mathrm{d} \ln K / \mathrm{d} T=\Delta_{\mathrm{r}} H / R T^{2}$

( $p$ constante) (5)

que integrada resulta em:

$\ln K=-\left[\Delta_{\mathrm{r}} H / R\right](1 / T)+C$

$(p$ constante) $(6)$ forma $y=a x+b(x=1 / T)$, ou seja, a equação de uma reta. $\mathrm{O}$ gráfico, como o da Figura 4, correspondente ao sistema ${ }^{12}$

$\mathrm{CaCO}_{3}(\mathrm{~s})=\mathrm{CaO}(\mathrm{s})+\mathrm{CO}_{2}(\mathrm{~g})$

em que $K^{\theta}=p\left(\mathrm{CO}_{2}, \mathrm{~g}\right) / p^{\theta}$, representa a variação linear de $\ln K^{\theta}$ com o inverso da temperatura $(1 / T)$, sendo possível calcular $\Delta_{\mathrm{r}} H(T)$ e $\Delta_{\mathrm{r}} S(T)$ da respectiva reação.

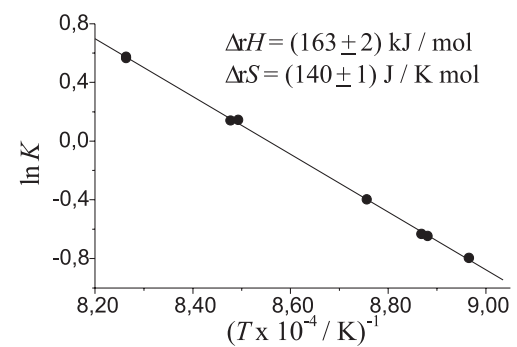

Figura 4. Diagrama de Van't Hoff, $\ln K(1 / T)$, correspondente ao sistema $\mathrm{CaCO}_{3}(\mathrm{~s})=\mathrm{CaO}(\mathrm{s})+\mathrm{CO}_{2}(\mathrm{~g})$, na faixa de temperatura 1113 a $1213 \mathrm{~K}$. Construído com dados da ref. 12

A equivalência de ambos os diagramas pode ser facilmente demonstrada. Os diagramas de Ellingham baseiam-se na Equação 1. Dividindo-a por $1 / R T$ (obviamente $T>0$, pela $2^{\mathrm{a}}$ Lei da Termodinâmica $\left.{ }^{5,13}\right)$ tem-se:

$-\frac{\Delta_{\mathrm{r}} G(T)}{R T}=-\frac{\Delta_{\mathrm{r}} H(T)}{R T}+\frac{\Delta_{\mathrm{r}} S(T) T}{R T}$

( $p$ constante) $(8)$

O primeiro membro dessa equação é igual a ln $K$, é a relação conhecida por "lei do equilíbrio químico" ou "isóbara de Van’t Hoff"

$\Delta_{\mathrm{r}} G(T)=-R T \ln K$

( $p$ constante) (9)

O primeiro termo do segundo membro da Equação 8 é igual ao termo correspondente da Equação 6 e o segundo termo é a respectiva constante de integração. Os dois diagramas permitem, utilizando-se dados experimentais de $\Delta_{\mathrm{r}} G(T)$ ou de $\ln K$, a diversas temperaturas, obter $\Delta_{\mathrm{r}} H(T)$ e $\Delta_{\mathrm{r}} S(T) . \Delta_{\mathrm{r}} G(T)$ pode ser obtido de medidas de força eletromotriz de células galvânicas (pilhas eletroquímicas), $E$, através da conhecida equação fundamental da Eletroquímica ${ }^{5,11}$ :

$\Delta_{\mathrm{r}} G(T)=-v\left(\mathrm{e}^{-}\right) F E$

( $p$ constante) (10)

sendo $v\left(\mathrm{e}^{-}\right)$o coeficiente estequiométrico dos elétrons nas equações químicas balanceadas dos dois lados da célula. A constante de equilíbrio termodinâmica $K$ pode ser obtida também de experimentos onde se pode medir concentrações (e daí as atividades) ou pressões parciais (e daí as fugacidades) do sistema em equilíbrio. O uso do diagrama de Ellingham ou de Van't Hoff passa a ser decorrente dos dados experimentais obtidos. A Figura 5 apresenta, de forma comparativa, os dois diagramas.

Apesar da equivalência de ambos, nem sempre se obtêm os mesmos resultados, devido à precisão dos dados experimentais e aos valores numéricos que serão ajustados à equação da reta. Este ajuste pode ser feito utilizando-se planílhas eletrônicas (Origin, Sigmaplot etc.), através do método dos mínimos quadrados (correlação linear).

Vamos ver dois exemplos, utilizando-se dados da literatura bem distantes entre si, para mostrar o paralelismo de ambos os diagramas. $\mathrm{O}$ primeiro deles refere-se à dissociação do iodo em fase gasosa:

$\mathrm{I}_{2}(\mathrm{~g})=2 \mathrm{I}(\mathrm{g})$

$(p=0,1 \mathrm{MPa})(11)$ 


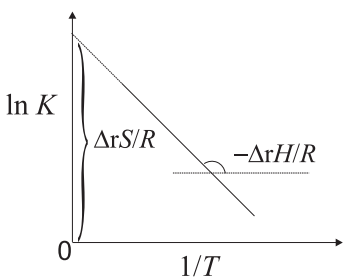

Diagrama de Van't Hoff

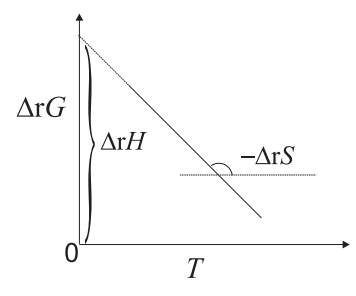

Diagrama de Ellingham
Figura 5. Diagramas de Van't Hoff e de Ellingham mostrados de forma comparativa

estudada por Starck e Bodenstein ${ }^{14}$, em 1910, medindo-se a pressão do sistema a diversas temperaturas e depois calculando-se $K$. Utilizamos a faixa de 1073 a $1473 \mathrm{~K}$ (5 pontos). Os correspondentes diagramas de Van't Hoff e Ellingham estão nas Figuras 6 e 7 e na Tabela 2 estão os valores encontrados. $\Delta_{\mathrm{r}} G$ foi calculado pela Equação 9. Note que os valores de $\Delta_{\mathrm{r}} H(T)$ e $\Delta_{\mathrm{r}} S(T)$ são concordantes dentro dos desvios estimados. A pequena diferença entre os valores dos desvios é devido ao ajuste.

Tabela 2. Valores de $\Delta_{\mathrm{r}} H^{\theta}$ e de $\Delta_{\mathrm{r}} S^{\theta}$ obtidos através dos diagramas de Van't Hoff ( $\mathrm{VH})$ e Ellingham (El) nos dois sistemas indicados. Todos os valores indicados são em $\mathrm{kJ} \mathrm{mol}^{-1}$

\begin{tabular}{lcccc}
\hline Sistema & $\Delta_{\mathrm{r}} H^{\theta}(\mathrm{VH})$ & $\Delta_{\mathrm{r}} H^{\theta}(\mathrm{El})$ & $\Delta_{\mathrm{r}} S^{\theta}(\mathrm{VH})$ & $\Delta_{\mathrm{r}} S^{\theta}(\mathrm{El})$ \\
\hline $\mathrm{I}_{2}(\mathrm{~g})=2 \mathrm{I}(\mathrm{g})$ & $154,2 \pm 0,2$ & $154,5 \pm 1,0$ & $106,3 \pm 0,8$ & $106,5 \pm 0,8$ \\
$(\mathrm{p}=0,1 \mathrm{MPa})$ & & & & \\
$\mathrm{Ru}_{(\mathrm{s})+\mathrm{O}_{2}(\mathrm{~g})=}$ & $-301 \pm 1$ & $-302 \pm 1$ & $-169 \pm 1$ & $-169 \pm 1$ \\
$\mathrm{RuO}_{2}(\mathrm{~s})$ & & & & \\
$(\mathrm{p}=0,1 \mathrm{MPa})$ & & & & \\
\hline
\end{tabular}

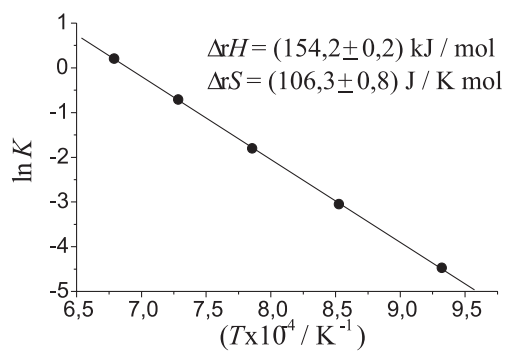

Figura 6. Diagrama de Van't Hoff para o sistema $I_{2}(g)=2 I(g)(p=0,1$ MPa), na faixa de 1073 a $1473 \mathrm{~K}$. Os valores de $\Delta r \mathrm{H} e \Delta r \mathrm{~S}$ foram calculados pela Equação 6 (vide também Tabela 2). Construído com dados da ref. 14

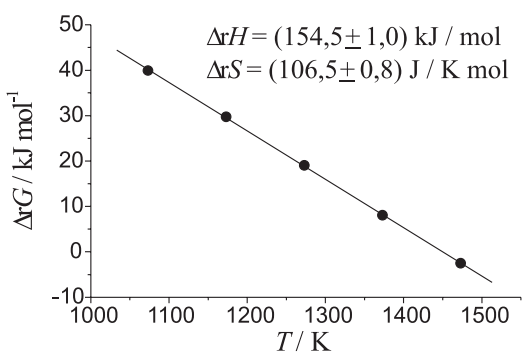

Figura 7. Diagrama de Ellingham para o sistema $I_{2}(g)=2 I(g)(p=0,1$ MPa), na faixa de 1073 a $1473 \mathrm{~K}$. Os valores de $\Delta r \mathrm{G}(\mathrm{T})$ foram calculados pela Equação 9 e os de $\Delta r \mathrm{H} e \Delta r \mathrm{~S}$, pela Equação 1 (vide também Tabela 2). Construído com dados da ref. 14

O segundo exemplo é a formação do óxido de rutênio:

$\mathrm{Ru}(\mathrm{s})+\mathrm{O}_{2}(\mathrm{~g})=\mathrm{RuO}_{2}(\mathrm{~s})$

$(p=0,1 \mathrm{MPa})(12)$ estudada por Cordfunke e Konings ${ }^{15}$, em 1988, que construíram uma célula galvânica baseada nesta reação e mediram a força eletromotriz da mesma, juntamente com a pressão de oxigênio, a diversas temperaturas. Utilizamos a faixa de 961,8 a $1056,7 \mathrm{~K}$ (5 pontos). Os correspondentes diagramas de Van't Hoff e Ellingham estão nas figuras 8 e 9 e os valores encontrados, na Tabela 2. Pela Equação 10 calculou-se $\Delta_{\mathrm{r}} G$ e pela Equação $9, \ln K$. Note que os valores de $\Delta_{\mathrm{r}} H(T)$ e $\Delta_{\mathrm{r}} S(T)$ são também concordantes.

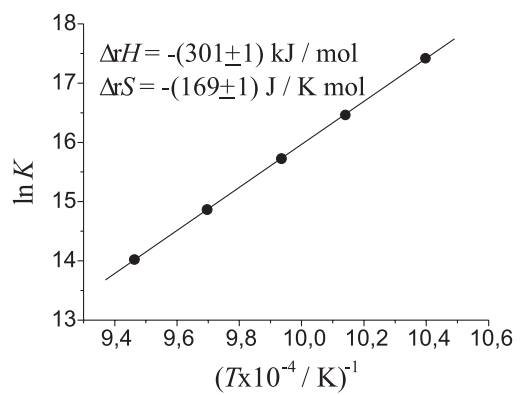

Figura 8. Diagrama de Van't Hoff para o sistema $\mathrm{Ru}(\mathrm{s})+\mathrm{O}_{2}(\mathrm{~g})=\mathrm{RuO}_{2}(\mathrm{~s})(\mathrm{p}$ $=0,1 \mathrm{MPa}$ ), na faixa de 961,8 a 1056,7 K. Os valores de $\ln \mathrm{K}$ foram calculados pela Equação 9 e os de $\Delta r \mathrm{H}$ e $\Delta r \mathrm{~S}$, pela Equação 1 (vide também Tabela 2). Construído com dados da ref. 15

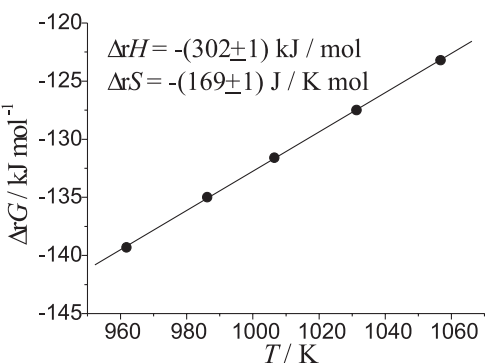

Figura 9. Diagrama de Ellingham para o sistema $\mathrm{Ru}(\mathrm{s})+\mathrm{O}_{2}(\mathrm{~g})=\mathrm{Ru} \mathrm{O}_{2}(\mathrm{~s})(\mathrm{p}=$ 0,1 MPa), na faixa de 961,8 a 1056,7 K. Os valores de $\Delta r \mathrm{H} e \Delta r \mathrm{~S}$ foram calculados pela Equação 1 (vide também Tabela 2). Construído com dados da ref. 15

\section{CONCLUSÃO}

Apesar de conhecidos há muito tempo, os diagramas de Van't Hoff e de Ellingham são equivalentes e complementares. Na literatura científica e técnica tradicionalmente utiliza-se um ou outro e o uso de ambos pode ser desnecessário na maior parte das vezes. Entretanto, a comparação dos dois diagramas certamente ajudará aos professores e estudantes, em suas atividades de ensino e aprendizagem, a compreenderem melhor certos aspectos da Termodinâmica Química.

\section{SÍMBOLOS UTILIZADOS}

$\Delta_{\mathrm{r}} X \equiv \sum_{\mathrm{B}} v_{\mathrm{B}} X$, sendo $v_{\mathrm{B}}$ o coeficiente estequiométrico da espécie $\mathrm{B}$, associado à equação química genérica $0=\sum_{\mathrm{B}} v_{\mathrm{B}} \mathrm{B}$.

$X$ = funções termodinâmicas de estado: $G, H, S, S T, C_{p}$.

$G=$ função de Gibbs ou energia livre de Gibbs.

$H=$ entalpia.

$S=$ entropia.

$C_{p}=$ capacidade calorífica molar à pressão constante.

$T=$ temperatura termodinâmica.

$p=$ pressão.

$p^{\theta}=$ pressão padrão $=0,1 \mathrm{MPa}$. 
$K=$ constante de equilíbrio termodinâmica.

$E=$ força eletromotriz de uma célula galvânica.

$F=$ constante de Faraday $=96485 \mathrm{C} / \mathrm{mol}$.

\section{REFERÊNCIAS}

1. Ellingham, H. J. T.; J. Soc. Chem. Ind. (London) 1944, 63, 125.

2. DeHof, R. T.; Thermodynamics in Materials Science, McGraw-Hill: New York, 1993.

3. Lupis, C. H. P.; Chemical Thermodynamics of Materials, North-Holland and Elsevier: New York, 1983.

4. Atkins, P. W.; Physical Chemistry, $6^{\text {th }}$ ed., Oxford: Oxford, 1998; Shriver, D. F.; Atkins, P. W.; Inorganic Chemistry, $3^{\text {rd }}$ ed., Oxford University Press: Oxford, 1999; Shriver, D. F.; Atkins, P. W.; Química Inorgânica, $3^{\text {rd }}$ ed., Bookman: Porto Alegre, 2006.

5. Chagas, A. P.; Termodinâmica Química, Ed. Unicamp: Campinas, 1999.
6. Put, P. J. van der; Inorganic Chemistry of Materials, Plenum Press: New York, 1998

7. Swaddle, T. W.; Inorganic Chemistry, Academic Press: San Diego (CA), 1997.

8. Maia, A. de S.; Osorio, V. K. L.; Quim. Nova 2003, 26, 595.

9. McGlashan, M. L.; Chemical Thermodynamics, Academic Press: London, 1979.

10. Chase Jr., M. W.; Davies, C. A.; Downey Jr., J. R.; Frurip, D. J.; McDonald, R. A.; Syverud, A. N.; JANAF Thermochemical Tables, $3^{\text {rd }}$ ed., J. Phys. Chem. Ref. Data 1985, 14, suppl. 1.

11. DeVoe, H.; Thermodynamics and Chemistry, Prentice Hall: Upper Saddle River (NJ), 2001.

12. Smyth, F. H.; Adams, L. H.; J. Am. Chem. Soc. 1923, 45, 1167.

13. Denbigh, K.; The Principles of Chemical Equilibrium, $4^{\text {th }}$ ed., Cambridge University Press: Cambridge, 1981.

14. Stark, G.; Bodenstein, M.; Z. Elektroch. 1910, 16, 961.

15. Cordfunke, E. H. P.; Konings, R. J. M.; Thermochim. Acta 1988, 129, 63. 